\title{
NONNEGATIVE AND SKEW-SYMMETRIC PERTURBATIONS OF A MATRIX WITH POSITIVE INVERSE
}

\author{
GIUSEPPE BUFFONI
}

\begin{abstract}
Let $A$ be a nonsingular matrix with positive inverse and $B$ a nonnegative matrix. Let the inverse of $A+v B$ be positive for $0 \leq v<v^{*}<+\infty$ and at least one of its entries be equal to zero for $v=v^{*}$; an algorithm to compute $v^{*}$ is described in this paper. Furthermore, it is shown that if $A+A^{\mathrm{T}}$ is positive definite, then the inverse of $A+v\left(B-B^{\mathrm{T}}\right)$ is positive for $0 \leq v<v^{*}$.
\end{abstract}

\section{INTRODUCTION}

Let

$$
A+v B
$$

be an $n \times n$ real matrix, where $A$ is a nonsingular matrix with positive inverse $([5,2,1]), B(B \neq 0)$ a nonnegative matrix and $v$ a nonnegative real parameter,

$$
A^{-1}>0, \quad B \geq 0, \quad B \neq 0, \quad v \geq 0 .
$$

The parameter $v$ may be considered as a measure of the size of the nonnegative perturbation $v B$ of the matrix $A$. Let

$$
Z(v)=(A+v B)^{-1}=\left[z_{i j}(v)\right] .
$$

For $v=0$, we have $Z(0)=A^{-1}>0$; thus, $\operatorname{det}(A+v B) \neq 0$ and $Z(v)>0$ in a sufficiently small neighborhood of 0 . This paper addresses the problem of finding the largest, possibly infinite, number $v^{*}$ such that $A+v B$ is nonsingular and $Z(v)>0$ in $\left[0, v^{*}\right)$. We will describe an algorithm (the iterative process (6)) to compute $v^{*}$ if $v^{*}<+\infty$. In the case $v^{*}=+\infty$, the successive approximations defined by (6) form a sequence diverging monotonically to $+\infty$.

We shall consider also matrices of the type

$$
C(v)=A+v\left(B-B^{\mathrm{T}}\right) ;
$$

here the matrix $A$ is perturbed by a skew-symmetric matrix which may be written as $B-B^{\mathrm{T}}$ with $B \geq 0$. It will be shown that if $A+A^{\mathrm{T}}$ is positive definite, then $C^{-1}(v) \geq Z(v)>0$ in $\left[0, v^{*}\right)$, where $Z$ is defined by (3).

Received July 26, 1988; revised November 23, 1988 and January 18, 1989.

1980 Mathematics Subject Classification (1985 Revision). Primary 65F30. 
Numerical calculations have been performed by using the matrix involved in the discrete analog of the integro-differential equation

$$
\frac{\partial u}{\partial t}=\frac{\partial}{\partial x}\left[p \frac{\partial u}{\partial x}\right]+q\left[u_{0}-u\right]+v \int_{0}^{1} K\left(x, x^{\prime}\right)\left[u_{0}\left(x^{\prime}\right)-u\left(x^{\prime}\right)\right] d x^{\prime}
$$

with boundary conditions $u(0)=u(1)=0$, where $p(x)>0, q(x) \geq 0$, $u_{0}(x) \geq 0$, and $K\left(x, x^{\prime}\right) \geq 0$. Equation (5) is a model for a spatially distributed community whose migration has both a random and a special deterministic component; more complicated models ( $n$-species communities, nonlinear) can be obtained including birth-death processes, competition and predator-prey interactions [4]. A direct finite difference approach to (5) provides a discrete approximation $\mathbf{u}$ of the steady state solution $u$ satisfying an equation of the type $(A+v B) \mathbf{u}=\mathbf{f} \geq 0$, where $A+v B$ is of type (2); the positivity of its inverse assures the positivity and the stability of $\mathbf{u}$.

\section{THE INVERSE OF $A+v B$}

Lemma 1. Assume (2) and let $\operatorname{det}(A+v B) \neq 0$ and $Z(v)>0$, with $Z(v)$ as defined in (3). Then $Z^{\prime}(v)<0$ and $Z^{\prime \prime}(v)>0$.

Proof. From the identity $(A+v B) Z(v)=I$ we obtain

$$
Z^{\prime}=-Z B Z, \quad Z^{\prime \prime}=-2 Z B Z^{\prime}=2 Z B Z B Z,
$$

where $Z^{\prime}=d Z / d v=\left[z_{i j}^{\prime}\right]$ and $Z^{\prime \prime}=d Z^{\prime} / d v=\left[z_{i j}^{\prime \prime}\right]$. As $B \geq 0, B \neq 0$, and $Z(v)>0$, there follows $Z^{\prime}(v)<0$ and $Z^{\prime \prime}(v)>0$.

Lemma 2. Under the assumptions of Lemma 1 , let $v_{\alpha}$ be the largest number such that $\operatorname{det}(A+v B) \neq 0$ in the interval $\left[0, v_{\alpha}\right)$. Then, either $v_{\alpha}=+\infty$, or an element of $Z(v)$ must change sign in $\left[0, v_{\alpha}\right)$.

Proof. As $v \longrightarrow v_{\alpha}$, at least one entry of $Z(v)$ must become infinite. Otherwise, in any interval $\left[0, v_{\beta}\right)$ where $Z(v)>0$ we have $Z^{\prime}(v)<0$ (Lemma 1); therefore, $Z(v)$ is bounded in $\left[0, v_{\beta}\right)$,

$$
0<Z(v) \leq Z(0)=A^{-1} .
$$

It follows that $v^{*}=\max v_{\beta} \leq v_{\alpha}$, with strict inequality if $v^{*}<+\infty$, because $0 \leq Z\left(v^{*}\right) \leq A^{-1}$. When $v^{*}<+\infty$, the thesis follows from $Z^{\prime}\left(v^{*}\right) \leq 0$ and Lemma 1 (note that the entries $z_{i j}(v)$ cannot vanish identically).

Theorem 1. Let $v^{*}$ be the largest, possibly infinite, number such that $Z(v)>0$ in $\left[0, v^{*}\right)$. Then $v^{*}$ is the limit of the sequence $\left\{v_{k}\right\}$ given by

$$
v_{k+1}=v_{k}+\min _{i, j: w_{k i j}>0} z_{k i j} / w_{k i j}, \quad k=0,1,2, \ldots, n ; v_{0}=0,
$$

where $Z_{k}=Z\left(v_{k}\right)=\left[z_{k i j}\right], W_{k}=-Z^{\prime}\left(v_{k}\right)=Z_{k} B Z_{k}=\left[w_{k i j}\right]$.

Proof. Let $v_{i j}^{*}$ be the smallest value of $v$ for which $z_{i j}(v)=0$, if such a value exists, or $+\infty$ otherwise. We have $v^{*}=\min _{i, j} v_{i j}^{*}$. In $\left[0, v^{*}\right)$, the matrix $Z(v)$ does not have singularities (Lemma 2) and its entries are strictly 
decreasing and convex functions of $v$ (Lemma 1). These regularity conditions on the entries $z_{i j}(v)$ allow us to obtain the sequence $\left\{v_{k}\right\}$, given by (6), as follows: we compute the Newton steps for the elements of the equation $Z(v)=$ 0 and use the smallest of them to update $v$.

The first iteration, with starting value $v_{0}=0$, produces the equations $z_{i j}(0)+$ $v z_{i j}^{\prime}(0)=0$, where $z_{i j}(0)>0$ and $z_{i j}^{\prime}(0)<0$. The smallest solution of these equations is the first approximation $v_{1}$ in (6) and it is the largest value of $v$ for which

$$
Z(0)+v Z^{\prime}(0)=A^{-1}-v A^{-1} B A^{-1} \geq 0 .
$$

As $Z(v)>Z(0)+v Z^{\prime}(0)$ for $0<v<v^{*}$, we have $v_{1}<v_{i j}^{*}, i, j=$ $1,2, \ldots, n$; therefore, $0<v_{1}<v^{*}$ and $Z_{1}>0, W_{1}>0$.

The successive approximations $v_{k}$ are defined as follows. Suppose we have computed the approximation $v_{k}$, for some $k>0$, for which we have $0<$ $v_{k}<v^{*}, Z_{k}>0, W_{k}>0$. We compute the Newton steps starting from the value $v_{k}$, common to all the equations $z_{i j}(v)=0$; this produces the equations $z_{i j}\left(v_{k}\right)+\left(v-v_{k}\right) z_{i j}^{\prime}\left(v_{k}\right)=0$. The approximation $v_{k+1}$ (the smallest solution of these equations) is the largest value of $v$ for which

$$
Z\left(v_{k}\right)+\left(v-v_{k}\right) Z^{\prime}\left(v_{k}\right)=Z_{k}-\left(v-v_{k}\right) W_{k} \geq 0
$$

and it is given by (6). As $Z(v)>Z\left(v_{k}\right)+\left(v-v_{k}\right) Z^{\prime}\left(v_{k}\right)$ for $v_{k}<v<v^{*}$, we have $v_{k+1}<v_{i j}^{*}, i, j=1,2, \ldots, n$; therefore $v_{k}<v_{k+1}<v^{*}$ and $Z_{k+1}>0, W_{k+1}>0$. We conclude that the sequence $\left\{v_{k}\right\}$ is increasing, bounded from above by $v^{*}$ if $v^{*}<+\infty$, and convergent to $v^{*}$ (note that $\left\{v_{k}\right\}$ cannot converge to a limit $v_{1}^{*}<v^{*}$ since this would imply $\left(v_{k+1}-v_{k}\right) \longrightarrow$ $\left.\min _{i j} z_{i j}\left(v_{1}^{*}\right) /\left|z_{i j}^{\prime}\left(v_{1}^{*}\right)\right|>0\right)$.

When $v^{*}=+\infty$, all the entries $z_{i j}(v)$ are positive, strictly decreasing, and convex functions of $v \in[0,+\infty)$ (the only possible solution of each equation $z_{i j}(v)=0$ is $\left.v^{*}=+\infty\right)$. If the sequence $\left\{v_{k}\right\}$ were bounded, then it would be convergent: $v_{k} \rightarrow v_{1}^{*}<+\infty$; as above, we would have $\left(\dot{v}_{k+1}-v_{k}\right) \rightarrow$ constant $>$ 0 . Thus, $\left\{v_{k}\right\}$ is not bounded and it is diverging monotonically to $+\infty$.

Remarks. (a) It is possible to show that the sequence $\left\{v_{k}^{\prime}\right\}$ given by

$$
v_{k+1}^{\prime}=v_{k}^{\prime}+\min _{i, j ; w_{0 i j}>0} z_{k i j} / w_{0 i j}, \quad k=0,1,2, \ldots ; v_{0}^{\prime}=0,
$$

is convergent to $v^{*}$, if $v^{*}<+\infty$, or divergent to $+\infty$ otherwise.

(b) Only for very small $n$ (the first few integers) can we obtain the analytic expressions of the entries $z_{i j}(v) \quad(i, j=1,2, \ldots, n)$ and find their zeros to evaluate $v^{*}$. The application of the iterative process (6) involves the numerical computation of the inverses $Z_{k}$, and each iteration requires $O\left(n^{3}\right)$ operations; however, the method has been applied successfully with $n$ equal to 30, 40, and 50 (for example, by using matrices from one-dimensional boundary value problems). 
(c) We can show the quadratic convergence [3, p. 260] of the process (6) when $v^{*}<+\infty$ and $Z^{\prime}\left(v^{*}\right)>0$. We introduce in (6) $z_{k i j}$ obtained from Taylor's formula

$$
z_{i j}\left(v^{*}\right)=z_{k i j}-\left(v^{*}-v_{k}\right) w_{k i j}+\frac{1}{2}\left(v^{*}-v_{k}\right)^{2} z_{i j}^{\prime \prime}\left(v_{k i j}\right),
$$

where $v_{k} \leq v_{k i j} \leq v^{*}$. After some manipulations we have

$$
v^{*}-v_{k+1}=\min _{i, j: w_{k i j}>0}\left[\frac{1}{2}\left(v^{*}-v_{k}\right)^{2} z_{i j}^{\prime \prime}\left(v_{k i j}\right)-z_{i j}\left(v^{*}\right)\right] / w_{k i j} ;
$$

thus, as $z_{i j}\left(v^{*}\right) \geq 0$ and $w_{k i j}>0$, it follows that

$$
s_{k+1} \leq \max _{i, j ; w_{k i j}>0} z_{i j}^{\prime \prime}\left(v_{k i j}\right) / w_{k i j} \rightarrow \max _{i, j} z_{i j}^{\prime \prime}\left(v^{*}\right) /\left|z_{i j}^{\prime}\left(v^{*}\right)\right|,
$$

where

$$
s_{k+1}=\left(v^{*}-v_{k+1}\right) /\left(v^{*}-v_{k}\right)^{2} .
$$

\section{THE INVERSE OF $C(v)=A+v\left(B-B^{\mathrm{T}}\right)$}

Theorem 2. Let the symmetric matrix $A+A^{\mathrm{T}}$ be positive definite. Then, in $\left[0, v^{*}\right)$ the spectral radius $h$ of the nonnegative matrix

$$
H(v)=v Z(v) B^{\mathrm{T}}
$$

is less than 1 , and $C^{-1}(v) \geq Z(v)>0$.

Proof. The matrix $C(v)$ given by (4) is now written as

$$
C(v)=(A+v B)[I-H(v)],
$$

where $H(v)$ is given by $(8)$. In $\left[0, v^{*}\right)$ we have $Z(v)>0$; it follows that $C^{-1}(v) \geq Z(v)>0$ if the spectral radius $h(v)=r(H)$ of the nonnegative matrix $H(v)$ is less than $1[5$, p. 83]. To the spectral radius $h$ there corresponds an eigenvector $\mathbf{u} \geq 0$; from the eigenvalue equation $v B^{\mathrm{T}} \mathbf{u}=h(A+v B) \mathbf{u}$ we obtain

$$
h=v \mathbf{u}^{\mathrm{T}} B \mathbf{u} /\left(\mathbf{u}^{\mathrm{T}} A \mathbf{u}+v \mathbf{u}^{\mathrm{T}} B \mathbf{u}\right) .
$$

We have $\mathbf{u}^{\mathrm{T}} A \mathbf{u}=\frac{1}{2}\left[\mathbf{u}^{\mathrm{T}}\left(A+A^{\mathrm{T}}\right) \mathbf{u}\right]>0$, because $A+A^{\mathrm{T}}$ is assumed positive definite. Thus, as $v \geq 0, \mathbf{u} \geq 0, B \geq 0$, it follows that $h<1$.

Remarks. By means of simple examples it is possible to show that:

(a) The condition $A+A^{\mathrm{T}}$ positive definite is not necessary to have $h(v)<$ $1,0 \leq v<v^{*}$.

(b) The condition $H(v) \geq 0,0 \leq v<v^{*}$, is not sufficient by itself to have $h(v)<1$.

\section{NUMERICAL RESULTS}

As a sample problem we use the matrix $A+v B$ obtained from a finite difference approximation to (5) using central differences and the trapezium rule. 
Here we present the results obtained by assuming in (5) that $p=1, q=0$, and $K\left(x, x^{\prime}\right)=\exp \left(-\left(x-x^{\prime}\right)^{2}\right)$ (sample problem 1). In this case, $A$ is a Stieltjes matrix [5, p. 85] and $B$ is a positive matrix. The inverses $Z_{k}$ are computed by means of the routine LINV2F of the IMSL Library. The results (double-precision computation) are shown in Table 1. The quantities $s_{k}$, given by (7), tend to a constant value confirming quadratic convergence. Values of $v$ greater than $v^{*}$, for which some computed entries of $Z(v)$ are less than zero are reported in the row $*$.

\section{TABLE 1}

Values of $v_{k}$ and of $s_{k}$ for the sample problem 1 for different values of the mesh spacing $1 / \mathrm{m}$.

\begin{tabular}{|c|c|c|c|c|c|}
\hline \multicolumn{2}{|c|}{$m=30$} & \multicolumn{2}{|c|}{$m=40$} & \multicolumn{2}{|c|}{$m=50$} \\
\hline$v_{k}$ & $s_{k}$ & $v_{k}$ & $s_{k}$ & $v_{k}$ & $s_{k}$ \\
\hline 0. & & 0. & & 0. & \\
\hline 5.145497 & 0.0658 & 5.076475 & 0.0678 & 5.035965 & 0.0690 \\
\hline 8.172000 & 0.0491 & 8.018864 & 0.0496 & 7.929456 & 0.0499 \\
\hline 8.820264 & 0.0505 & 8.633288 & 0.0510 & 8.524586 & 0.0517 \\
\hline 8.842959 & 0.0508 & 8.653839 & 0.0514 & 8.543958 & 0.0517 \\
\hline 8.842985 & 0.0508 & 8.653861 & 0.0513 & 8.543978 & 0.0516 \\
\hline 8.842985 & & 8.653861 & & 8.543978 & \\
\hline 8.85 & & 8.66 & & 8.55 & \\
\hline
\end{tabular}

TABLE 2

Values of $v_{k}$ and of $s_{k}$ for the sample problem 2 for different values of the mesh spacing $1 / \mathrm{m}$.

\begin{tabular}{|c|c|c|c|c|c|c|}
\hline \multirow[b]{2}{*}{$k$} & \multicolumn{2}{|c|}{$m=30$} & \multicolumn{2}{|c|}{$m=40$} & \multicolumn{2}{|c|}{$m=50$} \\
\hline & $v_{k}$ & $s_{k}$ & $v_{k}$ & $s_{k}$ & $v_{k}$ & $s_{k}$ \\
\hline 0 & 0 . & & 0 . & & 0 . & \\
\hline 1 & 0.411695 & 1.8439 & 0.299168 & 2.5543 & 0.234883 & 3.2660 \\
\hline 2 & 0.471457 & 0.2874 & 0.341517 & 0.3880 & 0.267634 & 0.4885 \\
\hline 3 & 0.472521 & 0.2899 & 0.342236 & 0.3912 & 0.268175 & 0.4924 \\
\hline 4 & 0.472521 & 0.2881 & 0.342236 & 0.3884 & 0.268175 & 0.5178 \\
\hline 5 & 0.472521 & & 0.342236 & & 0.268175 & \\
\hline$*$ & 0.48 & & 0.35 & & 0.27 & \\
\hline$* *$ & 0.49 & & 0.36 & & 0.28 & \\
\hline
\end{tabular}

Now we consider the matrix $C(v)=A+v\left(B-B^{\mathrm{T}}\right)$ obtained by assuming in (5) that $p=1, q=0$, and $K\left(x, x^{\prime}\right)=x-x^{\prime}$ (sample problem 2). Here the matrix $B$ is the nonnegative contribution due to $K\left(x, x^{\prime}\right)$ for $x \geq x^{\prime}$. The 
results are shown in Table 2. Values of $v$ greater than $v^{*}$, for which some computed entries of $Z(v)$ and of $C^{-1}(v)$ are less than zero are reported in the rows $*$ and $* *$, respectively. We note that $\left[0, v^{*}\right)$ is a sufficiently good approximation of the interval in which $C^{-1}(v)>0$.

\section{ACKNOWLEDGMENTS}

I wish to thank Professor Walter Gautschi and a referee for their criticism and suggestions.

\section{BIBLIOGRAPHY}

1. G. Buffoni and A. Galati, Matrici essenzialmente positive con inversa positiva, Boll. Un. Mat. Ital. (4) 10 (1974), 98-103.

2. K. Fan, Topological proofs for certain theorems on matrices with non-negative elements, Monatsh. Math. 62 (1958), 219-237.

3. J. R. Rice, Numerical methods, software and analysis, McGraw-Hill, 1983.

4. Y. M. Svirezhev and D. O. Logofet, Stability of biological communities, MIR, Moscow, 1983.

5. R. S. Varga, Matrix iterative analysis, Prentice-Hall, Englewood Cliffs, N. J. 1965.

ENEA CREa S. Teresa, C. P. 316, I-19100 La Spezia, Italy 